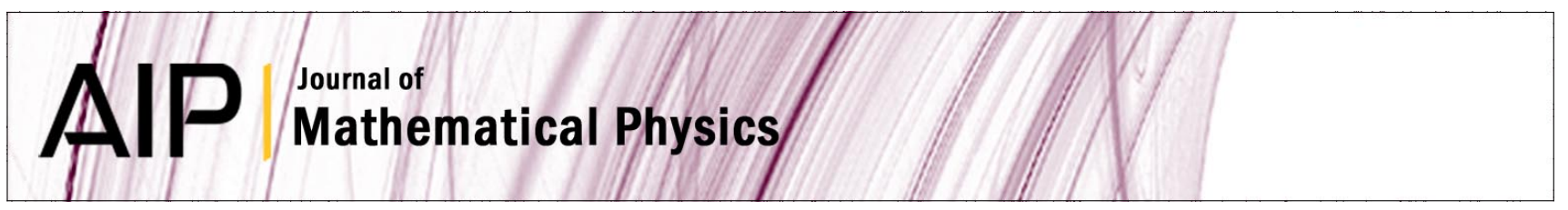

\title{
Coulomb-distorted plane wave: Partial wave expansion and asymptotic
} forms

I. Hornyak and A. T. Kruppa

Citation: J. Math. Phys. 54, 053502 (2013); doi: 10.1063/1.4803027

View online: http://dx.doi.org/10.1063/1.4803027

View Table of Contents: http://jmp.aip.org/resource/1/JMAPAQ/v54/i5

Published by the American Institute of Physics.

Additional information on J. Math. Phys.

Journal Homepage: http://jmp.aip.org/

Journal Information: http://jmp.aip.org/about/about_the_journal

Top downloads: http://jmp.aip.org/features/most_downloaded

Information for Authors: http://jmp.aip.org/authors

\section{ADVERTISEMENT}
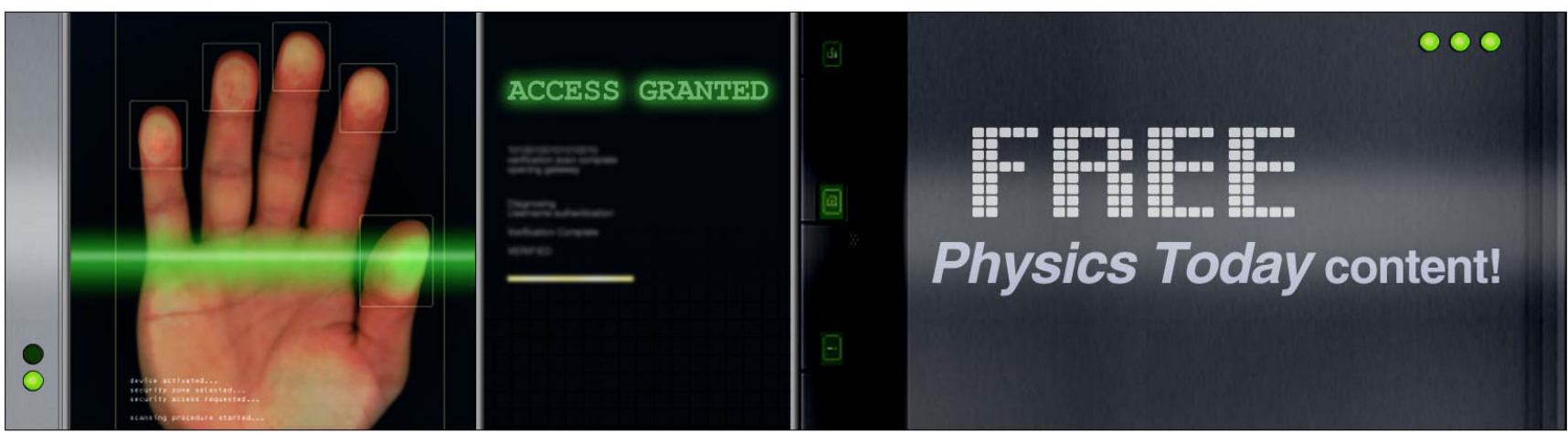


\title{
Coulomb-distorted plane wave: Partial wave expansion and asymptotic forms
}

\author{
I. Hornyak ${ }^{1, a)}$ and A. T. Kruppa 2, b) \\ ${ }^{1}$ University of Debrecen, Faculty of Informatics, PO Box 12, 4010 Debrecen, Hungary \\ ${ }^{2}$ Hungarian Academy of Sciences Institute for Nuclear Research, Bem tér 18/c, 4026 \\ Debrecen, Hungary
}

(Received 16 January 2013; accepted 11 April 2013; published online 1 May 2013)

\begin{abstract}
Partial wave expansion of the Coulomb-distorted plane wave is determined and studied. Dominant and sub-dominant asymptotic expansion terms are given and leading order three-dimensional asymptotic form is derived. The generalized hypergeometric function ${ }_{2} F_{2}(a, a ; a+l+1, a-l ; z)$ is expressed with the help of confluent hypergeometric functions and the asymptotic expansion of ${ }_{2} F_{2}(a, a ; a+l+1, a-l ; z)$ is simplified. (c) 2013 AIP Publishing LLC. [http://dx.doi.org/10.1063/1.4803027]
\end{abstract}

\section{INTRODUCTION}

Scattering of charged particles is an important and difficult topic in quantum mechanics. Even the two-body case is exceptional since it does not fit into the conventional time-dependent scattering theory approach. Several methods are proposed to overcome the difficulties caused by the long range behavior of the Coulomb interaction. In the time-dependent framework the formalism of Dollard ${ }^{1}$ modifies the Moller wave operator. In stationary approach the method of van Haaringen ${ }^{2}$ introduced Coulomb asymptotic states and Mulherin and Zinnes ${ }^{3}$ promoted distorted asymptotic states. The connection of the latter two formalism was also studied. ${ }^{4}$ Following Refs. 5 and 6 we will call the asymptotic state of Mulherin and Zinnes ${ }^{3}$ as Coulomb-distorted plane wave (CDPW).

The recently developed surface integral formalism of quantum scattering theory ${ }^{5-7}$ is valid not only for two-body scattering but it can be extended to scattering of three charged particles. Another advantage of this formalism is that it handles the short and long range interactions on an equal footing. It is said in Ref. 6 "All the results ... rely on the asymptotic forms of the plane wave and the Coulomb-distorted plane waves." The CDPW indeed plays an important role in the surface integral formalism. In this framework the full scattering wave function is composed of as a sum of the CDPW and a so-called scattered part. Furthermore, the surface integral expression of the scattering amplitude also refers to the CDPW.

A three-dimensional leading order asymptotic form of the CDPW was given in Ref. 5. Mathematical interpretation of this result was presented in Section 5 of Ref. 6. The asymptotic expansion can be interpreted in distributional sense and the properties of the considered test function space plays an important role. Based on our convergent partial wave (pw) expansion of the CDPW and the asymptotic form of the pw components we will derive a three-dimensional leading order asymptotic form for the CDPW. Our expression is in agreement with the result of Refs. 5 and 6 if the test function space given by Taylor ${ }^{8}$ is used.

Interestingly the pw expansion of the CDPW has been given only recently. ${ }^{9}$ In this work the pw components are applied for the description of scattering process of two particles based on the complex scaling method. Here we further study the pw decomposition and derive four different analytical forms. The asymptotic expansion of the pw component given in Ref. 9 contains a recursion relation for the expansion coefficients. In this paper we derive an explicit expression for them.

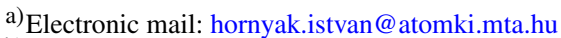

b) Electronic mail: kruppa.andras@atomki.mta.hu
} 
Our results concerning the CDPW are presented in Sec. II. Since the generalized hypergeometric function ${ }_{2} F_{2}(a, a ; a+l+1, a-l ; z)$ plays a substantial role in the formalism therefore in the Appendix the properties of this function are examined in detail. The summary is given in Sec. III.

\section{PARTIAL WAVE EXPANSION AND ASYMPTOTIC FORMS}

We write the pw expansion of the Coulomb-distorted plane wave in the form

$$
e^{i \mathbf{k r}}(k r \mp \mathbf{k r})^{ \pm i \gamma}=\sum_{l=0}^{\infty}(2 l+1) \tau_{l}^{( \pm)}(\gamma, k r) P_{l}(\cos (\vartheta)),
$$

where the upper and lower signs correspond to the post and prior form of the CDPW. The angle between the vectors $\mathbf{k}$ and $\mathbf{r}$ is signed by $\vartheta$ and the Legendre polynomial is denoted by $P_{l}(x)$. The real variable $\gamma$ in (1) is the Sommerfeld parameter and $\mathbf{k}$ is the momentum vector. The bold face letters $\mathbf{k}$ and $\mathbf{r}$ denote elements of the three-dimensional Euclidean space $\mathbb{R}^{3}$, while $k$ and $r$ denote the corresponding magnitudes. The radial function $\tau_{l}^{( \pm)}(\gamma, k r)$ can be calculated by the integral

$$
\tau_{l}^{( \pm)}(\gamma, k r)=\frac{1}{2}(k r)^{ \pm i \gamma} \int_{-1}^{1} e^{ \pm i k r x}(1 \mp x)^{ \pm i \gamma} P_{l}(x) d x
$$

A compact expression for the pw component $\tau_{l}^{( \pm)}(\gamma, k r)$ can be given for arbitrary $l$. Using (2) and the formula 2.17.5.6 in Ref. 10 we get

$$
\tau_{l}^{(+)}(\gamma, k r)=\frac{(-i \gamma)_{l}}{(1+i \gamma)_{l+1}}(2 k r)^{i \gamma} e_{2}^{i k r} F_{2}(1+i \gamma, 1+i \gamma ; l+2+i \gamma, 1+i \gamma-l ;-2 i k r)
$$

and

$$
\tau_{l}^{(-)}(\gamma, k r)=(-1)^{l} \frac{(i \gamma)_{l}}{(1-i \gamma)_{l+1}}(2 k r)^{-i \gamma} e_{2}^{-i k r} F_{2}(1-i \gamma, 1-i \gamma ; l+2-i \gamma, 1-i \gamma-l ; 2 i k r),
$$

where $(a)_{n}$ is the Pochhammer symbol and ${ }_{2} F_{2}$ is the generalized hypergeometric function. ${ }^{11}$ From the explicit forms (3) and (4) one can notice that $\tau_{l}^{(-)}(\gamma, k r)=(-1)^{l} \tau_{l}^{(+)}(\gamma, k r)^{*}$. In order to further study the function $\tau_{l}^{( \pm)}(\gamma, k r)$ the properties of the function ${ }_{2} F_{2}(a, a ; a+l+1, a-l ; z)$ are investigated in the Appendix. In the case of the post form of CDPW the following identifications can be made $a=1+i \gamma$ and $z=-2 i k r$ and in the prior form we have $a=1-i \gamma, z=2 i k r$. Using Theorem 1 of the Appendix and the formula (3) we can write down three equivalent expressions for the partial wave component

$$
\begin{gathered}
\tau_{l}^{(+)}(\gamma, k r)=\frac{e^{i k r+\gamma \pi / 2}}{2 i k r} \sum_{n=0}^{l}(-1)^{n}\left(\begin{array}{l}
l \\
n
\end{array}\right) \frac{(l+1)_{n}}{n !} \gamma(1+i \gamma+n, 2 i k r)(2 i k r)^{-n}, \\
\tau_{l}^{(+)}(\gamma, k r)=(-1)^{l}(2 k r)^{i \gamma} e^{i k r} \sum_{n=0}^{l}(-1)^{n}\left(\begin{array}{l}
l \\
n
\end{array}\right) \frac{(l+1)_{n}}{(1+i \gamma)_{n+1}}{ }_{1} \mathrm{~F}_{1}(1+i \gamma, 2+i \gamma+n ;-2 i k r),
\end{gathered}
$$

and

$$
\tau_{l}^{(+)}(\gamma, k r)=\frac{e^{\gamma \pi / 2}}{2 i k r}\left(e^{i k r} \kappa_{l}^{+}(1+i \gamma,-2 i k r)+e^{-i k r} \kappa_{l}^{-}(1+i \gamma,-2 i k r)\right) .
$$

In charge-less case (i.e., $\gamma=0$ ) the CDPW goes into an ordinary plane wave. Using Proposition 2 of the Appendix we get the expected result for the pw components, i.e., $\tau_{l}^{( \pm)}(0, k r)=i^{l} j_{l}(k r)$, i.e., the post and prior forms are identical.

Using Theorem 2 of the Appendix we get for the asymptotic expansion of $\tau_{l}^{( \pm)}(\gamma, k r)$ as $r \rightarrow \infty$

$$
\begin{aligned}
\tau_{l}^{(+)}(\gamma, k r) \sim & \frac{e^{i k r}}{2 i k r} e^{\gamma \pi / 2} \Gamma(1+i \gamma)_{3} F_{1}\left(1+i \gamma,-l, l+1 ; 1 ;(2 i k r)^{-1}\right) \\
& -\frac{e^{-i k r}}{2 i k r}(2 k r)^{i \gamma}(-1)^{l} \sum_{n=0}^{\infty} d_{n}^{(l)} \frac{1}{(-i k r)^{n}}
\end{aligned}
$$


and

$$
\begin{aligned}
\tau_{l}^{(-)}(\gamma, k r) \sim & -(-1)^{l} \frac{e^{-i k r}}{2 i k r} e^{\gamma \pi / 2} \Gamma(1-i \gamma)_{3} F_{1}\left(1-i \gamma,-l, l+1 ; 1 ;(-2 i k r)^{-1}\right) \\
& +\frac{e^{i k r}}{2 i k r}(2 k r)^{-i \gamma} \sum_{n=0}^{\infty}\left(d_{n}^{(l)}\right)^{*} \frac{1}{(i k r)^{n}} .
\end{aligned}
$$

The expansion coefficients $d_{n}^{(l)}$ satisfy the recursion (A20) and (A21) and the solution of the recursion is given by (A22). In these equations we have to identify $a$ by $1+i \gamma(1-i \gamma)$ for the post (prior) form of the pw component. The expressions (8) and (9) show both the dominant and sub-dominant terms and the nature of the terms are determined by the sign of $\operatorname{Im}(i k r)$. Instead of the study of the explicit form of $\tau_{l}^{( \pm)}(\gamma, k r)$ the asymptotic expansions (8) and (9) can be obtained directly from (2) with a straightforward but tedious calculation based on Theorem 227 of Ref. 18.

Now we derive a three-dimensional leading order asymptotic form for the CDPW. Substituting expressions (8) and (9) into (1) and keeping only the leading order terms we get our result in the form of a distribution

$$
e^{i \mathbf{k r}}(k r \mp \mathbf{k r})^{ \pm i \gamma} \sim \pm \frac{2 \pi}{i k r}\left(e^{ \pm i k r} e^{\gamma \pi / 2} \Gamma(1 \pm i \gamma) \delta(\hat{\mathbf{r}} \mp \hat{\mathbf{k}})-e^{\mp i k r}(2 k r)^{ \pm i \gamma} \delta(\hat{\mathbf{r}} \pm \hat{\mathbf{k}})\right) .
$$

It is known that the prior form of the CDPW can be simply obtained from the post form expression: one has to replace $\mathbf{k}$ with $\mathbf{- k}$ and make complex conjugation. According to (10) this procedure is valid also for the asymptotic forms. form $^{17}$

In charge-less case the three-dimensional asymptotic expression (10) goes into the well known

$$
e^{i \mathbf{k r}} \sim \frac{2 \pi}{i k r}\left(e^{i k r} \delta(\hat{\mathbf{r}}-\hat{\mathbf{k}})-e^{-i k r} \delta(\hat{\mathbf{r}}+\hat{\mathbf{k}})\right)
$$

On the test function spaces $D^{ \pm}$of Ref. 6 our result goes into Eqs. (197) and (198) of Ref. 6,

$$
e^{i \mathbf{k r}}(k r \mp \mathbf{k r})^{ \pm i \gamma} \sim \mp \frac{2 \pi}{i k r} e^{\mp i k r}(2 k r)^{ \pm i \gamma} \delta(\hat{\mathbf{r}} \pm \hat{\mathbf{k}}) \text { on } D^{ \pm}
$$

On test functions belong to $D^{ \pm}$the contribution of the first term of (10) is zero. Our rigorously derived result (10) differs from the expression of Refs. 5 and 6. However, the surface integral formalism, as explained in Ref. 6, depends only on the validity of (12).

\section{SUMMARY}

The generalized hypergeometric function ${ }_{2} F_{2}(a, a ; a+l+1, a-l ; z)$ is expressed as a finite sum of confluent hypergeometric functions. Three equivalent expressions are determined and so the numerical evaluation can be simplified. Dominant and sub-dominant terms are identified in the asymptotic expansion. A closed expression is given for the coefficients of the asymptotic expansion. Using the previous results four equivalent expressions are determined for the partial wave component of the Coulomb-distorted plane wave. The asymptotic expansion of the partial wave component is deduced. Rigorously derived leading order three-dimensional asymptotic form of the Coulomb-distorted plane wave is given.

\section{ACKNOWLEDGMENTS}

The publication was supported by the TÁMOP-4.2.2.C-11/1/KONV-2012-0001 project. The project has been supported by the European Union, co-financed by the European Social Fund. 


\section{APPENDIX: THE HYPERGEOMETRIC FUNCTION ${ }_{2} F_{2}(a, a ; a+I+1, a-l ; z)$}

We will express the function ${ }_{2} F_{2}(a, a ; a+l+1, a-l ; z)$ in terms of finite linear combination of confluent hypergeometric functions. In order to do this we need a proposition.

Proposition 1. Let $l$ be a non-negative integer and let $\mathbb{X}_{l}:=\{x \in \mathbb{Z} \mid x \leq l\}$. If $z \in \mathbb{C} \backslash\{0\}$ and $a \in \mathbb{C} \backslash \mathbb{X}_{l}$ then

$$
\sum_{k=0}^{l}(-1)^{k}\left(\begin{array}{l}
l \\
k
\end{array}\right)(l+1)_{k}(1-a)_{k}{ }_{1} \mathrm{~F}_{1}(-k, a-k ; z) \frac{z^{-k}}{k !}=(-1)^{l}{ }_{3} \mathrm{~F}_{1}(1-a,-l, l+1 ; 1 ;-1 / z) .
$$

Proof. The definition of the confluent hypergeometric function can be turned into the form

$$
{ }_{1} \mathrm{~F}_{1}(-k, a-k ; z)=\sum_{s=0}^{k}(-1)^{s}\left(\begin{array}{l}
k \\
s
\end{array}\right) \frac{z^{s}}{(a-k)_{s}} .
$$

Using this expression we get on the left-hand side of (A1)

$$
\sum_{k=0}^{l} \sum_{s=0}^{k}(-1)^{k+s}\left(\begin{array}{l}
l \\
k
\end{array}\right)\left(\begin{array}{l}
k \\
s
\end{array}\right) \frac{(l+1)_{k}(1-a)_{k}}{k !(a-k)_{s}} z^{s-k}
$$

We can rearrange the summation indexes and get

$$
(-1)^{l} \sum_{s=0}^{l}\left(\begin{array}{l}
l \\
s
\end{array}\right)(l+1)_{s}(1-a)_{s} \frac{z^{-s}}{s !} \varepsilon_{l}(s)
$$

where

$$
\varepsilon_{l}(s)=\sum_{k=0}^{l-s}(-1)^{k}\left(\begin{array}{c}
l-s \\
k
\end{array}\right) \frac{(l+1)_{l-k} s !}{(l+1)_{s}(l-k) !} .
$$

Using the formula 0.160 .2 of Ref. 12 we get $\varepsilon_{l}(s)=1$ and we can recognize that the summation in (A2) gives the function ${ }_{3} \mathrm{~F}_{1}(1-a,-l, l+1 ; 1 ;-1 / z)$.

Theorem 1. Let $l$ be a non-negative integer and let $z \in \mathbb{C} \backslash\{0\}, a \in \mathbb{C} \backslash \mathbb{Z}$ then the function ${ }_{2} F_{2}(a$, $a ; a+l+1, a-l ; z)$ can be given in the following forms:

$$
\begin{gathered}
(-1)^{l} \frac{(a)_{l+1}}{(1-a)_{l}} \sum_{k=0}^{l}(-1)^{k}\left(\begin{array}{l}
l \\
k
\end{array}\right) \frac{(l+1)_{k}}{(a)_{k+1}} 1 \mathrm{~F}_{1}(a, a+k+1 ; z), \\
(-z)^{-a} \frac{(a)_{l+1}}{(1-a)_{l}} \sum_{k=0}^{l}\left(\begin{array}{l}
l \\
k
\end{array}\right) \frac{(l+1)_{k}}{k !} \gamma(a+k,-z)(z)^{-k}, \\
\frac{(a)_{l+1}}{(1-a)_{l}}(-z)^{-a}\left(\kappa_{l}^{+}(a, z)+e^{z} \kappa_{l}^{-}(a, z)\right) .
\end{gathered}
$$

Here we introduced the notations

$$
\kappa_{l}^{-}(a, z)=(-1)^{l+1} \sum_{n=0}^{l} \frac{(l+n) !}{n !(l-n) !} \frac{(-1)^{n}}{z^{n}} U(-a+1,-a-n+1 ;-z)
$$

and

$$
\kappa_{l}^{+}(a, z)=\Gamma(a){ }_{3} F_{1}(a,-l, l+1 ; 1 ;-1 / z) .
$$

The confluent hypergeometric function of the second kind ${ }^{11}$ and the incomplete gamma function ${ }^{11}$ are signed by $U(a, b, z)$ and $\gamma(a, z)$, respectively. 
Proof. First we show that (A3) equals (A4). Using the identity 5.3.5.4 of Ref. 10 and the expression 7.11.3.1 of Ref. 10 we get for (A3)

$$
(-z)^{-a} \frac{(a)_{l+1}}{(1-a)_{l}} \sum_{k=0}^{l} \sum_{s=0}^{k}(-1)^{l+k+s}\left(\begin{array}{l}
l \\
k
\end{array}\right)\left(\begin{array}{l}
k \\
s
\end{array}\right) \frac{(l+1)_{k}}{k !} \gamma(a+s,-z)(-z)^{-s} .
$$

Rearranging the summation we have

$$
(-z)^{-a} \frac{(a)_{l+1}}{(1-a)_{l}} \sum_{s=0}^{l}(-1)^{s}\left(\begin{array}{l}
l \\
s
\end{array}\right) \frac{(l+1)_{s}}{s !} \gamma(a+s,-z)(-z)^{-s} \varepsilon_{l}(s)
$$

and using that $\varepsilon_{l}(s)=1$ we get (A4).

Next we show that ${ }_{2} F_{2}(a, a ; a+l+1, a-l ; z)$ equals (A4). Using the

$$
{ }_{p} F_{q}\left(\begin{array}{cc}
\left(a_{p-1}\right), & \sigma+l \\
\left(b_{q-1}\right), & \sigma
\end{array} \mid z\right)=\sum_{k=0}^{l}\left(\begin{array}{l}
l \\
k
\end{array}\right) \frac{z^{k}}{(\sigma)_{k}} \frac{\prod\left(a_{p-1}\right)_{k}}{\prod\left(b_{q-1}\right)_{k}} p_{-1} F_{q-1}\left(\begin{array}{c}
\left(a_{p-1}\right)+k \\
\left(b_{q-1}\right)+k
\end{array} \mid z\right)
$$

identity, which was proved in Ref. 13 , we get for ${ }_{2} F_{2}(a, a ; a+l+1, a-l ; z)$

$$
(-z)^{-a} \sum_{k=0}^{l} \sum_{s=0}^{l}(-1)^{k+s}\left(\begin{array}{l}
l \\
k
\end{array}\right)\left(\begin{array}{l}
l \\
s
\end{array}\right) \frac{(a)_{l+1}}{l !(a-l)_{k}} \gamma(a+k+s,-z)(-z)^{-s} .
$$

The following expression, valid for $k>0$, can be easily established using the formula 8.356 .9 of $^{12}$

$$
\gamma(b+k, z)=(b)_{k} \gamma(b, z)-e^{-z} z^{b} \sum_{m=0}^{k-1} \frac{(b)_{k}}{(b)_{m+1}} z^{m}
$$

and for $k=0$ we have $\gamma(b+0, z)=(b)_{0} \gamma(b, z)=\gamma(b, z)$. Choosing $b=a+s$ in (A10) and substituting it into (A9) we get

$$
\begin{aligned}
& (-z)^{-a} \frac{(a)_{l+1}}{(1-a)_{l}} \sum_{s=0}^{l}(-1)^{s}\left(\begin{array}{l}
l \\
s
\end{array}\right) \frac{(l+1)_{s}}{s !} \gamma(a+s,-z)(-z)^{-s}+ \\
& -\frac{(a)_{l+1}}{l !} e^{z} \sum_{k=1}^{l} \sum_{m=0}^{k-1}(-1)^{k}\left(\begin{array}{l}
l \\
k
\end{array}\right) \frac{\Gamma(a+k) \Gamma(l+m+1-k)(-z)^{m}}{(a-l)_{k} \Gamma(l+a+m+1)} \frac{1}{\Gamma(-[k-1-m])} .
\end{aligned}
$$

In the derivation of this expression we used 0.160 .2 of Ref. 12 . We have to consider the case $a \in \mathbb{C} \backslash \mathbb{Z}$ and $l \geq k \geq 1$ so we have that $k-1-m$ is a non-negative integer. We get that the double sum in (A11) is zero and this means that ${ }_{2} F_{2}(a, a ; a+l+1, a-l ; z)$ is identical with (A4).

Finally we show that (A5) equals (A3). Applying the expression 7.2.2.2 of Ref. 10 we get for (A5)

$$
\frac{(a)_{l+1}}{(1-a)_{l}}\left(\sum_{n=0}^{l}(-1)^{n+l}\left(\begin{array}{l}
l \\
n
\end{array}\right) \frac{(l+1)_{n}}{(a)_{n+1}}{ }_{1} \mathrm{~F}_{1}(a, a+1+n ; z)+(-z)^{-a} \Gamma(a) \epsilon_{l}(a, z)\right),
$$

where

$$
\begin{aligned}
\epsilon_{l}(a, z)= & { }_{3} F_{1}(a,-l, l+1 ; 1 ;-1 / z) \\
& -(-1)^{l} \sum_{n=0}^{l}(-1)^{n}\left(\begin{array}{l}
l \\
n
\end{array}\right)(l+1)_{n}(a)_{n}{ }_{1} \mathrm{~F}_{1}(-n,-a+1-n ; z) \frac{z^{-n}}{n !} .
\end{aligned}
$$

According to Proposition $1 \epsilon_{l}(a, z)=0$ and so we derived (A3) from (A5).

In order to show that the pw component of CDPW is proportional to the spherical Bessel function in the case of charge-less particles we will need the following statement. 
Proposition 2. Let $l$ be a non-negative integer and let $z \in \mathbb{C} \backslash\{0\}$ and $a \in \mathbb{C} \backslash \mathbb{Z}$ then

$$
\lim _{a \rightarrow 1}(1-a)_{l 2} F_{2}(a, a ; a+l+1, a-l ; z)=i^{l}(l+1) ! e^{z / 2} j_{l}(i z / 2) .
$$

Proof. It is easy to establish that $(1)_{l+1} e^{z} \kappa_{l}^{-}(1, z) / z=-i^{l}(l+1) ! e^{z / 2} h_{l}^{(2)}(i z / 2) / 2$ and $(1)_{l+1} \kappa_{l}^{+}(1, z) / z=-i^{l}(l+1) ! e^{z / 2} h_{l}^{(1)}(i z / 2) / 2$ using 10.1.16 and 10.1.17 of Ref. 11. From these facts and from (A5) the claim follows.

Theorem 2. Let $l \geq 0$ integer, $a \in \mathbb{C}, 0<\operatorname{Re}(a)<l+2$ then the asymptotic expansion of the function ${ }_{2} F_{2}(a, a ; a+l+1, a-l ; z)$ at $|z| \rightarrow \infty$ can be written in the form

$$
\begin{aligned}
& { }_{2} F_{2}(a, a ; a+l+1, a-l ; z) \sim \frac{(a)_{l+1}}{(1-a)_{l}}(-z)^{-a} \kappa_{l}^{+}(a, z)+ \\
& (-1)^{l} \frac{(a)_{l+1}}{(1-a)_{l}} \frac{e^{z}}{z} \sum_{n=0}^{\infty} \frac{(1-a)_{n}(a)_{l}}{(a-n)_{l}}{ }_{3} F_{2}\left(\begin{array}{ccc}
-l, & -l, & -n \\
1, & 1-a-l & 1
\end{array}\right) \frac{1}{z^{n}} .
\end{aligned}
$$

Proof. We will use the expression (A5) to determine the asymptotic expansion. First we notice that $\kappa_{l}^{+}(a, z)$ is a finite sum, it is a polynomial of order $l$ in the variable $1 / z$. This means that $\frac{(a)_{l+1}}{(1-a)_{l}}(-z)^{-a} \kappa_{l}^{+}(a, z)$ can be considered as an asymptotic expansion at $|z| \rightarrow \infty$ with respect to the asymptotic sequence ${ }^{14} z^{-a} / z^{n}$. The function $\kappa_{l}^{-}(a, z)$, according to Eq. (A6), is a finite linear combination of confluent hypergeometric functions of second kind and since the asymptotic expansion of $U(a, b, z)$ is given by 15.5.2 of Ref. 11 we can properly rearrange the summation indexes and we can write

$$
\kappa_{l}^{-}(a, z) \sim(-1)^{l} \frac{(-z)^{a}}{z} \sum_{n=0}^{\infty}\left(\sum_{m=0}^{\min (n, l)}(-1)^{m} \frac{(l+m) !}{m !(l-m) !} \frac{(m+1)_{n-m}(1-a)_{n-m}}{(n-m) !}\right) \frac{1}{z^{n}} .
$$

Using the formulas 7.2.3.15 and 7.3.5 of Ref. 10 we can carry out the summation over $m$ on the right-hand side of (A15) and get

$$
\kappa_{l}^{-}(a, z) \sim(-1)^{l} \frac{(-z)^{a}}{z} \sum_{n=0}^{\infty} \frac{(1-a)_{n}(a)_{l}}{(a-n)_{l}}{ }_{3} F_{2}\left(\begin{array}{ccc|c}
-l, & -l, & -n \\
1, & 1-a-l & 1
\end{array}\right) \frac{1}{z^{n}} .
$$

The expressions (A14) shows that if $\operatorname{Im}(z)>0$ than the dominant term is given by the second term of the right-hand side of (A14) and if $\operatorname{Im}(z)<0$ then the roles of the terms in (A14) interchange.

The complete asymptotic expansion of the function ${ }_{2} F_{2}\left(a_{1}, a_{2} ; b_{1}, b_{2} ; z\right)$ was given by Luke in Chapter 5.11.3 of Ref. 15. However in our case the numerator parameters are equal to each others and we cannot use this result. We rewrite the function ${ }_{2} F_{2}(a, a ; a+l+1, a-l ; z)$ in terms of Meijer's G function (see 5.11.1(1) in Ref. 15),

$$
{ }_{2} F_{2}(a, a ; a+l+1, a-l ; z)=\frac{(-1)^{l}(a)_{l+1}}{(1-a)_{l}} G_{2,3}^{1,2}\left(-z \mid \begin{array}{ccc}
1-a, & 1-a \\
0, & -a-l, & l+1-a
\end{array}\right) .
$$

For the asymptotic expansion of the Meijer's G function we can use formula 5.10(10) of Ref. 15 and if we apply 5.7.(13-15) and 5.9.2 of Ref. 15 we get

$$
{ }_{2} F_{2}(a, a ; a+l+1, a-l ; z) \sim-\frac{(-1)^{l}(a)_{l+1}}{(1-a)_{l}} H_{2,2}(-z) .
$$

Taking into account 5.11.1(18) and 5.11.3(4) in Ref. 15 we can write

$$
H_{2,2}(-z)=-\frac{e^{z}}{z} \sum_{n=0}^{\infty} d_{n}^{(l)} 2^{n} \frac{1}{z^{n}} .
$$


The coefficients $d_{n}^{(l)}$ satisfy the recurrence relation (see 5.11.3(6) in Ref. 15)

$$
4(n+1) d_{n+1}^{(l)}=2\left(2 n^{2}-n(2 a-3)-l^{2}-l-a+1\right) d_{n}^{(l)}-n(n-l-a)(n+l+1-a) d_{n-1}^{(l)}
$$

with initial conditions (see 5.11.1(18-20) in Ref. 15)

$$
d_{0}^{(l)}=1, \quad d_{1}^{(l)}=\frac{1}{2}\left(1-l^{2}-l-a\right) .
$$

The asymptotic expansion (A18) is valid in the region $-\frac{\pi}{2}+\delta \leq \arg (-z) \leq \frac{3 \pi}{2}-\delta$ where $\delta>0$.

We will show that our asymptotic expansion (A14) and formula (A18) are identical in the considered $z$ region. Among the two terms at the right-hand side of (A14) the second one is the dominant in the considered $z$ region and the first one can be neglected. To rewrite (A18) and (A19) we have to solve the recurrence relation (A20).

Theorem 3. Let $l$ and $n$ be non-negative integers and let $a \in \mathbb{C}, 0<\operatorname{Re}(a)<l+2$ then the solution of the recurrence relation (A20) with initial conditions (A21) is

$$
d_{n}^{(l)}=\frac{(1-a)_{n}(a)_{l}}{2^{n}(a-n)_{l}}{ }_{3} F_{2}\left(\begin{array}{ccc}
-l, & -l, & -n \\
1, & -a-l+1 & 1
\end{array}\right) .
$$

Proof. We prove this theorem by induction. With direct calculation it is easy to check that the claim is satisfied for $n=0,1,2$. Assuming that (A22) is true for $n=s$ and $n=s+1$ the claim, after some trivial simplification, is reduced to the validity of the equation

$$
\begin{aligned}
(s+2)(s+2-l-a)_{3} F_{2}\left(\begin{array}{ccc}
-l, & -l, & -s-2 \mid \\
1, & -a-l+1 & 1
\end{array}\right)= \\
=\left[2(s+1)^{2}-(s+1)(2 a-3)-l^{2}-l-a\right]_{3} F_{2}\left(\begin{array}{ccc}
-l, & -l, & -s-1 \mid \\
1, & -a-l+1
\end{array}\right) \\
\quad-(s+1)(s+2+l-a)_{3} F_{2}\left(\begin{array}{ccc|c}
-l, & -l, & -s & 1 \\
1, & -a-l+1 & 1
\end{array}\right) .
\end{aligned}
$$

This equation, however, is known to be true. ${ }^{16}$

Using Theorem 3 and Eq. (A19) we see that (A18) and the second term on the right-hand side of (A14) are indeed identical.

${ }^{1}$ J. D. Dollard, J. Math. Phys. 5, 729 (1964).

${ }^{2}$ H. van Haaringen, J. Math. Phys. 17, 995 (1976).

${ }^{3}$ D. Mulherin and I. I. Zinnes, J. Math. Phys. 11, 1402 (1970).

${ }^{4}$ R. O. Barrachina and J. J. Macek, J. Math. Phys. 30, 2581 (1989).

${ }^{5}$ A. S. Kadyrov, I. Bray, A. M. Mukhamedzhanov, and A. T. Stelbovics, Phys. Rev. A 72, 032712 (2005).

${ }^{6}$ A. S. Kadyrov, I. Bray, A. M. Mukhamedzhanov, and A. T. Stelbovics, Ann. Phys. (N.Y.) 324, 1516 (2009).

${ }^{7}$ I. Bray, D. V. Fursa, A. S. Kadyrov, A. T. Stelbovics, A. S. Kheifets, and A. M. Mukhamdedzhanov, Phys. Rep. 520, 135 (2012).

${ }^{8}$ J. R. Taylor, Il Nuovo Cimento B 23, 313 (1974).

${ }^{9}$ I. Hornyak and A. T. Kruppa, Phys. Rev. A 85, 022702 (2012).

${ }^{10}$ A. P. Prudnikov, Y. A. Brychkov, and O. I. Marichev, Integrals and Series (Gordon and Breach Science Publishers, New York, 1990).

${ }^{11}$ Handbook of Mathematical Functions, edited by M. Abramowitz and I.A Stegun (Dover, New York, 1964).

${ }^{12}$ Table of Integrals, Series, and Products, 7th ed., edited by I. S. Gradshteyn and I. M. Ryzhik (Elsevier/Academic Press, San Diego, 2007).

${ }^{13}$ P. W. Karlsson, J. Math. Phys. 12, 270 (1971).

${ }^{14}$ A. Erdélyi, Asymptotic Expansions (Dover Publications, New York, 1956).

${ }^{15}$ Y. L. Luke, The Special Functions and Their Approximations (Academic Press, San Diego, 1969), Vol. I.

${ }^{16} \mathrm{See}$ http://functions.wolfram.com/07.27.17.0001.01 for recurrence identity for ${ }_{3} \mathrm{~F}_{2}$.

${ }^{17}$ A. Messiah, Quantum Mechanics (Dover Publications, Mineola, 1999).

${ }^{18}$ L. Sirovich, Techniques of Asymptotic Analysis (Springer-Verlag, New York, 1971). 